\title{
Philonsorbonne
}

$10 \mid 2016$

Année 2015-2016

Matière et philosophie première. À propos du livre $\mathrm{H}$ de la Métaphysique d'Aristote

\section{Pierre-Marie MOREL}

\section{Q OpenEdition}

Journals

Édition électronique

URL : https://journals.openedition.org/philonsorbonne/819

DOI : 10.4000/philonsorbonne.819

ISSN : 2270-7336

Éditeur

Publications de la Sorbonne

\section{Édition imprimée}

Date de publication : 1 janvier 2016

Pagination : 153-168

ISSN : 1255-183X

\section{Référence électronique}

Pierre-Marie MOREL, « Matière et philosophie première. À propos du livre $\mathrm{H}$ de la Métaphysique d'Aristote », Philonsorbonne [En ligne], 10 | 2016, mis en ligne le 20 janvier 2016, consulté le 08 juin 2021. URL : http://journals.openedition.org/philonsorbonne/819; DOI : https://doi.org/10.4000/ philonsorbonne.819 


\title{
Matière et philosophie première. À propos du livre H de la Métaphysique d'Aristote
}

\author{
Pierre-Marie MOREL \\ Université Paris 1 Panthéon-Sorbonne - UMR 7219 Gramata \\ Institut Universitaire de France
}

La matière a le mauvais rôle dans la Métaphysique d'Aristote. Parce que les traités qui composent cette dernière mènent l'enquête sur les premières causes et les premiers principes et cherchent à définir une science de l'être en tant qu'être, et parce que celui-ci est d'abord et essentiellement substance et forme, l'étude de la matière et de la causalité matérielle n'a guère de place, à première vue, en philosophie première ${ }^{1}$. La critique des théories antérieures, au livre $\mathrm{A}$, conduit en outre à rejeter les thèses de ceux qui privilégient la cause matérielle - ou l'idée qu'ils se font de la matière - et négligent, ou ignorent, les autres types de déterminations causales. Lorsque l'on quitte le contexte dialectique à proprement parler et que l'on aborde l'enquête positive sur l'ousia en tant que telle, en particulier dans le livre Z, les choses se présentent en principe différemment: on ne part plus, comme dans le livre $\mathrm{A}$, des conceptions erronées de la matière; on se trouve face à ce que l'on peut identifier, globalement, comme la doctrine même

\footnotetext{
1. Je parlerai ici de "philosophie première» au sens large, comme c'est majoritairement l'usage, pour désigner la science dont relèvent les traités métaphysiques dans leur ensemble, et sans réduction à un éventuel axe proprement théologique ni à l'examen d'un type d'étant particulier. Voir en ce sens l'étude récente de G. GUYOMARC'H, «Fonctions et objets de 'philosophie première' chez Aristote ", Revue de Philosophie Ancienne, 2014/2, p. 137-178. Plusieurs amis, étudiants et collègues m'ont fait bénéficier de leurs remarques lorsque j'ai présenté les versions préparatoires de cet article à Bergame, Bogotá, Mexico, Rome et Venise, et parmi eux, plus particulièrement, Maddalena Bonelli, Riccardo Chiaradonna, Alfonso Correa, Francesco Fronterotta, Francesca Masi, Carlo Natali, Ricardo Salles, Simone Seminara, Franco Trabattoni. Je tiens à les en remercier.
} 
d'Aristote $^{2}$. La matière est alors nettement distinguée de l'ousia véritable, le chapitre $3 \mathrm{du}$ livre $\mathrm{Z}$ refusant à la matière le statut de substance, parce qu'elle n'est qu'un substrat indéterminé, alors que l'ousia se caractérise avant tout par le fait qu'elle est quelque chose de séparé et constitue un « ceci », un existant par soi particulier :

[1] Si l'on examine les choses à partir de ce point de vue, il suit que la matière est substance. Or c'est impossible, car on est d'avis que le séparé et le ceci appartiennent au premier titre à la substance, c'est pourquoi on sera d'avis que la forme et le composé des deux <i.e. : forme et matière> sont substance plutôt que la matière.

Métaph., Z, 3, 1029a26-30

Toutefois, une lecture plus attentive montre que la matière n'est pas totalement exclue de la substantialité. La question est débattue, et cela d'autant plus qu'elle pose le problème de l'objet même de la Métaphysique, ou tout au moins des livres dits « centraux » $(\mathrm{Z}, \mathrm{H}, \Theta)$ : si l'enquête sur la substance a pour objet premier la substance en tant que forme, c'est-à-dire ce dont il y a définition à proprement parler ${ }^{3}$, alors la caractérisation par la matière ne saurait y occuper qu'une position marginale. On peut du reste en dire autant de l'étude du devenir. ${ }^{4}$. C'est d'ailleurs pourquoi un certain nombre de spécialistes de la Métaphysique ont considéré que les chapitres de $\mathrm{Z}$ qui traitent directement de la genesis $(\mathrm{Z}, 7-9)$ n'appartenaient pas à la première rédaction de $Z$, mais avaient été insérés après coup. Je ne discuterai pas ici cette question sans doute insoluble, mais je note que le problème de fond concerne également le court livre $\mathrm{H}$, non seulement parce qu'il est étroitement lié à $\mathrm{Z}$, ainsi que l'indique clairement $\mathrm{H} 1$, mais également parce qu'il fait une place importante à la matière et à l'analyse du devenir 5 .

2. Je n'aborderai pas ici la question de savoir dans quelle mesure et jusqu'à quel point le livre $\mathrm{Z}$ doit être considéré comme un traité dialectique, dans l'idée qu'il serait en dialogue constant avec Platon et la doctrine des formes intelligibles. Cette position est défendue par S. MenN, "Metaphysics Z10-16 and the Argument-Structure of Metaphysics Z », Oxford Studies in Ancient Philosophy, v. 21, Winter 2001, p. 83-134. J'admets que Z est en partie un traité dialectique, mais il ne fait guère de doute qu'il contient également un grand nombre de thèses proprement aristotéliciennes, notamment sur la substance, et qu'il a un caractère beaucoup plus positif que l'enquête du livre A sur les causes.

3. Comme l'a par exemple soutenu M. FredE, notamment dans « The Definition of Sensible Substances in Metaphysics Z », in Devereux D., Pellegrin P. (éd.), Biologie, logique et métaphysique chez Aristote, Actes du Séminaire C.N.R.S.-N.S.F., Oléron, 28 juin-3 juillet 1987, Paris, 1990, p. 113-129. Je reviendrai plus bas sur ce point, à propos de H2, que Frede ne prend pas en compte.

4. Voir en ce sens M. Burnyeat, A Map of Metaphysics Zeta, Pittsburg, Mathesis Publications, 2001.

5. C'est un des aspects de la lecture du livre H que je propose dans P.-M. Morel, Aristote. Métaphysique, Livre H (êta), Traduction, introduction et commentaire, Paris, Vrin, 2015. 
Je me concentrerai précisément sur la façon dont la matière est abordée en $\mathrm{H}$, tout au moins dans les passages qui me paraissent les plus significatifs. Ce texte montre en effet qu'il n'y a pas d'incohérence, dans la Métaphysique, à dire que la matière est substance, même si elle l'est seulement en un sens ou jusqu'à un certain point. Le fait qu'elle ne remplisse pas toutes les conditions pour être désignée comme ousia, ainsi que le montre Z3, ne signifie pas que l'on doive exclure la matière, définitivement et sous tout rapport, de l'ousia des substances composées. Je n'entends pas dire ici que la matière est en quelque sorte intégrée à la forme ${ }^{6}$, à la substance formelle, mais plutôt qu'elle a part, en corrélation avec la forme, à ce qui fait que la substance composée est, précisément, substance. En d'autres termes, la matière ne peut pas être la substance d'une entité composée de matière et de forme, elle n'est jamais par elle-même substance au sens absolu, mais elle n'est pas pour autant exclue de la substantialité, c'est-à-dire de ce qui fait qu'une substance composée donnée est une substance. La thèse que je veux défendre est précisément que l'objet de $\mathrm{H}-$ ou l'un de ses principaux objets - est de confirmer et d'évaluer cette implication de la matière dans la substantialité.

Ce parcours suppose que l'on adopte deux hypothèses de travail : la première porte sur le lien entre $\mathrm{Z}$ et $\mathrm{H}$; la seconde sur la lecture de $\mathrm{H}$ luimême. Concernant le premier point, et en m'appuyant sur une lecture de H1 que j'ai proposée ailleurs ${ }^{7}$, je considère que $\mathrm{H} 1$, tout en résumant en partie les acquis de $\mathrm{Z}$, annonce, en introduisant le livre $\mathrm{H}$, une enquête qui doit apporter des éléments complémentaires par rapport à $Z$. En second lieu, j'estime que le livre $\mathrm{H}$, malgré son désordre relatif, n'est pas aussi chaotique qu'on l'a parfois pensé. On invoque notamment les discontinuités observables dans le chapitre 3 et l'apparente autonomie du chapitre 6 par rapport aux chapitres précédents. Sur ce dernier point, je ne dirais pas qu'il y a une parfaite continuité dans l'argumentation globale du livre $\mathrm{H}$, mais il me semble que le principe de charité peut être appliqué plus généreusement qu'on ne le fait généralement, si l'on admet que les résultats du chapitre 6 sont préparés par les chapitres précédents.

On sait en effet que la fin de H6 affirme deux thèses conjointes à propos de la matière :

(a) l'équivalence de la matière et de la puissance : [2] «d'une part la matière et d'autre part la forme, d'un côté ce qui est en puissance et de l'autre ce qui est en acte ${ }^{8}$;

(b) l'unité de la matière ultime ou prochaine (eschatê) et de la forme dans le composé : [3] « la matière prochaine et la forme sont une seule et même chose, l'une en puissance et l'autre en acte $)^{9}$.

\footnotetext{
6. Comme l'a soutenu en substance M. Peramatzis, Priority in Aristotle's Metaphysics, Oxford, Oxford University Press, 2011.

7. Voir mon commentaire de $\mathrm{H}$, cité ci-dessus.

8. H, 6, 1045a23-24.
} 
Quelles que soient les difficultés que ces deux assertions posent en elles-mêmes, elles justifient à mon sens une grande partie des développements antérieurs du livre $\mathrm{H}$. Les deux thèses de $\mathrm{H} 6$ ne peuvent être atteintes, en effet, que parce que la notion même de matière a fait l'objet d'une nouvelle élaboration. Les observations successives de $\mathrm{H}$ en posent les bases ${ }^{10}$.

Après avoir brièvement rappelé la situation de la problématique de la matière en $\mathrm{Z}$, je montrerai que $\mathrm{H} 1$ met l'accent sur la substance sensible composée de matière et de forme - et donne de ce fait à la matière une importance nouvelle. Il s'agit notamment d'expliquer en quel sens la matière est « elle aussi substance ». Aristote établit en outre trois choses à propos de la matière : (a) qu'elle intervient dans la définition des substances sensibles ; (b) qu'elle contribue à l'analyse causale, non pas seulement par progression à partir des composants, mais également en direction des composants (l'analyse peut donc être régressive aussi bien que progressive); (c) que la matière comprend différents degrés de détermination, dès lors que l'on identifie une matière propre de la chose. La notion de matière ainsi élaborée permet de définir des niveaux de potentialité, dont le dernier, selon H6, est substantiellement uni à la forme elle-même ${ }^{11}$.

$* * *$

Il n'est pas nécessaire de revenir sur le détail de l'argumentation du livre A de la Métaphysique contre les physiciens qui ont eu le tort de penser que la matière était l'unique principe de toutes choses. En A, 3, 983b6-18, Aristote emploie un argument radical, selon lequel cette thèse a pour conséquence de rendre inconcevable toute génération et toute corruption. Si tout est matière, en effet, et si la matière est inengendrée et incorruptible parce qu'elle est elle-même l'origine de toute chose et le substrat permanent du devenir, alors on doit conclure qu'il n'y a rien qui puisse être engendré ou détruit. Ce n'est assurément pas le dernier mot d'Aristote sur ce point, car il reconnaît à ses prédécesseurs le mérite d'avoir eu une sorte de prénotion du substrat. Cette prénotion est susceptible, certes, de nous égarer, mais également de nous mettre sur la bonne voie.

Par ailleurs, dans le passage cité, ce qui est pris comme exemple de substrat permanent, ce n'est pas un élément matériel, comme l'eau ou le feu, mais Socrate, en tant que sujet de «beau» ou de «musicien». Ainsi, en

\section{H, 6, 1045b17-19.}

10. Voir en ce sens M. BURNYEAT, op. cit., p. 69, à propos de H : « Aristotle's reworking of the form-matter contrast, in terms of the more general notions of actuality and potentiality, continues all through $\mathrm{H}$ and comes to a climax in H6. » Burnyeat semble même considérer plus loin (p. 71) que H6 est le point culminant de l'enquête engagée en Z1. S. MENN, op. cit., rattache pour sa part H à Z17 - plutôt qu'à Z dans son ensemble -, séquence qui constituerait selon lui la doctrine positive sur le logos tês ousias.

11. Voir en ce sens M.L. GiLl, Aristotle on Substance. The Paradox of Unity, Princeton, Princeton University Press, 1989, p. 128 sq. 
évoquant la cause matérielle et en la qualifiant en 983 b10 de «substance

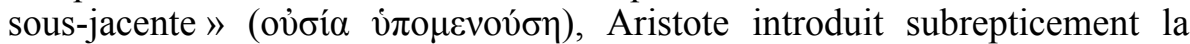

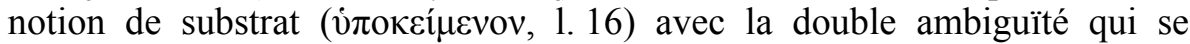
trouve débattue en Z3 : la matière, qui remplit cette fonction, est-elle pour autant ousia? Le substrat doit-il s'entendre uniquement comme sujet matériel indéterminé, ou bien doit-on aussi l'entendre comme sujet logique de prédication ${ }^{12}$, substance première au sens des Catégories $^{13}$ ?

En Z, la question « qu'est-ce que la substance ?» conduit à envisager, comme on sait, quatre possibilités : l'être essentiel ou quiddité, l'universel, le genre, le substrat ou sujet. L'examen de la quatrième possibilité conduit logiquement à rappeler la position de ceux qui font de la matière le substrat de toutes choses. Aristote rejette toutefois l'identification de la substance à la matière-sujet, comme on l'a vu dans le texte [1].

Ce texte pose plusieurs difficultés qui tiennent pour partie à son caractère partiellement dialectique. Si la notion de matière est abordée, ce n'est pas au cours d'une argumentation intégralement positive, mais en revenant aux théories antérieures. Se pose en outre la question de savoir si la «matière » dont il est ici question est la même « matière » que celle dont on parlera en d'autres occurrences : il se peut qu'Aristote parle ici de la matière la plus indéterminée, au moins la matière de niveau élémentaire, par opposition à la matière ultime évoquée par exemple en H6 et qui ne fait qu'un avec la forme et l'acte. Je propose de laisser pour l'instant cette question de côté, pour m'en tenir à une observation purement factuelle : ce passage dit expressément, et littéralement, qu'il est impossible que la matière soit substance.

L'affaire va se présenter différemment dans la suite du livre Z. C'est le cas en $Z, 7-9^{14}$, en particulier, où l'étude porte sur les choses en devenir, ce qui conduit nécessairement à examiner la matière dont elles sont composées. Cet examen conduit à formuler sérieusement l'hypothèse d'une présence de la matière dans la définition. En Z, 7, 1033a4-5, Aristote affirme clairement que «le cercle de bronze a la matière dans sa définition». Comment comprendre "matière" dans ce contexte ? Plusieurs commentateurs - le Pseudo-Alexandre, Bonitz, ou encore Tricot dans les notes de sa traduction estiment qu'il s'agit du genre (la forme circulaire), qui est matière des différences. Celles-ci spécifieraient le cercle de bronze. Cependant il y a

12. Ambiguïté rappelée en Z, 13, 1038b5-6.

13. Catég., 5, 2a11-14.

14. Sur cette section, je renvoie notamment au livre à paraître de S. MENN, aimablement communiqué par l'auteur, The Aim and the Argument of Aristotle's Metaphysics (unplublished version). Il estime que Z7-9 appartient originellement à $Z$ et s'insère dans l'enquête sur la substance formelle. Il relie en effet l'objet de Z7-9 à la question de l'essence comme principe, comprenant cette séquence comme « ... an investigation of the conditions of coming-to-be, in order to assess whether the fact that $X$ has come-to-be requires that the essence of $X$ be a previously existing archê ». Il estime également qu'elle participe de la réfutation de la doctrine des formes intelligibles : «we need not posit Platonic forms to account for comingto- be ». Menn pense du reste que H, 3, 1043b13-21 fait référence à cette section. 
aussi de bonnes raisons de penser qu'il est ici question de la matière sensible - celle qui entre dans la composition de la substance sensible -, c'est-à-dire le bronze ${ }^{15}$. La seconde interprétation peut poser problème si l'on s'en tient à la lettre de plusieurs passages, comme Z, 10, 1035b331036a1, qui semble exclure absolument la matière de la définition au sens rigoureux. La suite immédiate du passage, en Z, 7, 1033a5 sq., va toutefois dans ce sens : Aristote montre qu'il faut, lorsqu'on énonce la matière, faire

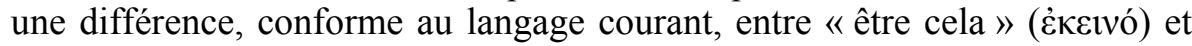

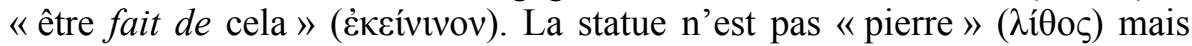
« de pierre » ( $\lambda i \operatorname{ivvo\zeta ).~Il~s'agit~donc~clairement~de~la~matière~sensible~ou~}$ physique. Sauf à considérer qu'il n'y a aucune continuité entre les deux moments du texte, on peut donc supposer que, dans le cercle de bronze de la phrase précédente, c'est bien de la matière sensible qu'il s'agit. Le passage de $\mathrm{Z7}$ invite en ce sens à envisager un type ou un niveau de matière suffisamment déterminé pour trouver place dans la définition des substances sensibles, considérées en tant que sensibles et en tant que la mention de leur matière est nécessaire et non pas accidentelle. En d'autres termes, la mention de la matière n'est pas nécessaire pour définir le cercle - qui peut être indifféremment de bronze ou de n'importe quelle autre matière, ou encore, comme figure géométrique, immatériel -, ni aucune réalité dont ni le genre ni la différence ultime ne sont d'ordre matériel, mais elle est probablement requise dans le cas inverse : le cercle de bronze se définira non seulement par son genre - cercle -, mais aussi par la différence qui tient à sa matière, le bronze.

Il ressort de ce très rapide survol de quelques passages cruciaux de $\mathrm{Z}$ que la matière n'est pas la substance, parce qu'elle n'est ni un tode $t i$, un « ceci », ni quelque chose de séparé, mais aussi qu'elle entre - au moins sous certaines conditions - dans la définition des êtres en devenir, et qu'elle présente différents degrés de détermination.

Le livre $\mathrm{H}$ va développer ces points et finalement conduire à la thèse selon laquelle l'unité du composé tient à l'unité immédiate de la matière et de la forme, parce que cette unité n'a pas de facteur supplémentaire en dehors de la matière et de la forme elles-mêmes, ni de cause externe, sinon l'action du moteur qui, dans la génération, fait passer de la puissance à l'acte $^{16}$.

\section{$* * *$}

Commençons par H1. Dans ce chapitre introductif, Aristote entend récapituler un certain nombre d'éléments considérés comme acquis. La

15. Voir en ce sens D. Ross, Aristotle's Metaphysics. Text with Introduction and Commentary, Oxford, Clarendon Press, 2 vol., 1924, 1958, 1966, vol. II, 185 ; M. BURNYEAT, op. cit., p. 36 ; D. Bostock, Aristotle. Metaphysics. Books $Z$ and $H$, translated with a Commentary, Oxford, Clarendon Press, 1994, p. 127 ; Notes on H- $\Theta$ of Aristotle's Metaphysics, being the record by M. Burnyeat and others, Oxford, 1984.

16. H, 6, 1045b20-22. 
discussion dialectique ne prend pas fin pour autant, puisqu'il revient sur les opinions dominantes concernant la substance. Sur certaines substances, dit-il, tous sont d'accord; sur d'autres - comme les idées et les réalités mathématiques - seuls quelques-uns le sont. Les substances qui font l'objet d'un consensus sont les substances sensibles, et c'est avec celles-ci que se termine le chapitre :

[4] Mais venons-en maintenant aux substances communément admises. Ce sont les substances sensibles; or les substances sensibles ont toutes une matière. Or est substance le sujet, qui en un sens est la matière (j'appelle «matière » ce qui, n'étant pas un ceci en acte, est un ceci en puissance), et en un autre sens la notion et la forme, ce qui, étant un ceci, est séparable en raison. Troisièmement, c'est ce qui est constitué < de matière et de forme>, et dont seul il y a génération et destruction, et qui est séparable sans restriction, car parmi les substances proprement définissables, les unes sont séparables mais pas les autres.

Métaph., H, 1, 1042a24-31

Ce passage fait écho à Z3 sur un point essentiel, à savoir les trois hypothèses concernant l'identité du substrat: la matière, la forme ou le composé des deux ${ }^{17}$. La convergence avec Z3 est donc claire, mais la différence ne l'est pas moins, puisque le texte de $\mathrm{H}$ introduit la distinction entre puissance et acte, ouvrant ainsi une perspective nouvelle, qui va être développée en H2 (1042b10; 1043a15 sq.); H5 (1044b30-36) : H6 $(1045 \mathrm{a} 24,32, \mathrm{~b} 19,21)$, puis traitée systématiquement en $\Theta^{18}$.

On constate également, et cette fois en désaccord littéral avec $Z$ - voir le texte [1] de Z3 -, que la matière n'est plus opposée au $\tau$ ó $\delta \varepsilon \tau 1$, puisqu'elle est un $\tau$ ó $\delta \varepsilon \tau \imath$ «en puissance ». C'est à n'en pas douter un tournant dans l'enquête sur la substance. Il est clair, dès lors, que le « résumé » de H1 est tout autre chose qu'un résumé, et qu'il est bien l'amorce d'une conclusion qui ne pouvait qu'être inattendue à la fin de $\mathrm{Z}$, et cela même si l'on prend en compte l'intérêt des chapitres 7-9 pour le devenir et les substances sensibles.

Les lignes suivantes vont cependant inviter à franchir un pas de plus, en affirmant littéralement que la matière « est substance » :

[5] Mais il est manifeste que la matière également est substance (ö $\tau \delta^{\prime}$

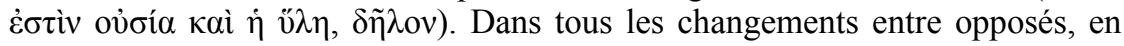
effet, il y a quelque chose qui est sujet pour les changements. Ainsi, selon le

\section{Z, 3, 1029a1-3.}

18. La continuité de $\mathrm{H}$ à $\Theta$ est discutée, et il serait imprudent de se prononcer trop vite sur l'économie générale des livres centraux, mais il y a selon moi des arguments forts en faveur d'un cheminement de $\mathrm{Z}$ à $\Theta$ qui passerait par $\mathrm{H}$, c'est-à-dire par la traduction du modèle hylémorphique en termes de puissance et d'acte. Voir en ce sens M. BURNYEAT, op. cit., p. 69 : «H, and then $\mathrm{ZH}$, expect $\Theta$ as their sequel ». Je renvoie une fois de plus à mon introduction dans P.-M. Morel, Aristote. Métaphysique, Livre H (êta)..., op. cit., p. 57-59. 
lieu : ce qui maintenant est ici et ensuite ailleurs ; selon l'augmentation, ce qui maintenant est de telle grandeur et ensuite plus petit ou plus grand; et selon la modification, ce qui maintenant est sain et ensuite malade. De même encore, dans le changement substantiel, ce qui maintenant est en train d'advenir et ensuite en état de destruction, et ce qui maintenant est sujet au sens du ceci et ensuite sujet par privation.

Métaph., H, 1, 1042a32-b3

La tension avec Z3, où l'on estimait « impossible » que la matière soit substance, est ici à son maximum. La suite du livre $\mathrm{H}$ ne va pas l'atténuer mais, bien au contraire, la renforcer, comme on peut en juger d'après les tournures « la substance entendue comme sujet au sens de matière » $(\dot{\eta} \mu \dot{\varepsilon} v$

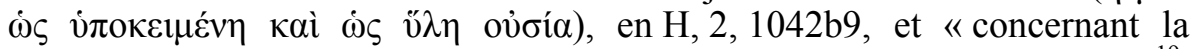

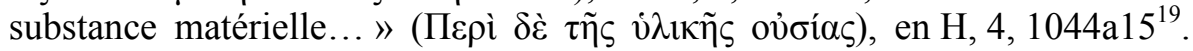
L'attribution à la matière du statut de substance n'est donc pas un accident, une expression proposée comme « en passant».

Il n'est toutefois pas nécessaire de dramatiser le contraste avec Z3 pour montrer l'importance de ces formulations. On peut en effet atténuer le conflit en notant que $\mathrm{Z3}$ ne nie pas totalement le caractère substantiel de la matière mais indique simplement que la matière n'est pas substance de plein droit ou encore qu'elle a le plus faible «coefficient» de substantialité, pour reprendre une expression suggérée par P. Pellegrin ${ }^{20}$. Ceux que critique Aristote ont le tort de croire que seule la matière est substance parce qu'ils considèrent un seul critère de substantialité, le sujet ultime ${ }^{21}$, et oublient les deux autres critères énoncés en 1029a27-28, à savoir le "séparé » et le « ceci ». Le texte de $\mathrm{H} 1$ peut vouloir dire que la matière est substance, mais qu'elle n'est pas la seule à l'être - d'où, sans doute, le кaì : " la matière également est substance »-, sans oublier qu'elle l'est avec une restriction de taille : elle n'est un « ceci » qu'en puissance. La matière n'est pas un « ceci » en acte et ne peut donc être substance au sens éminent. C'est du reste ce que laisse entendre le début de H2 : "Puisqu'il y a accord sur la substance entendue comme sujet et comme matière, et qu'elle est la substance en puissance, il reste à dire ce qu'est la substance, entendue comme acte, des choses sensibles » (1042b9-11). Pour résumer l'acquis principal de H1, la matière est " substance », mais elle l'est en tant que sujet du changement et constitue ainsi le « ceci » en puissance, et non pas en acte.

Néanmoins, l'insistance sur l'hypothèse " matière » est significative, de même que l'on doit sans doute tenir pour significative la brutalité de la formule. Elle résonne en effet comme une provocation et comme une annonce. Une provocation, puisque le texte de H1 s'adresse, ainsi que l'a

19. Voir aussi l'occurrence de cette expression que l'on trouve en $\Theta, 7,1049 \mathrm{a} 36$.

20. Voir P. Pellegrin, «Taxinomie, moriologie, division. Réponses à G.E.R. Lloyd», in Devereux D., Pellegrin P. (éd.), Biologie, logique et métaphysique chez Aristote, Actes du Séminaire C.N.R.S.-N.S.F., Oléron, 28 juin-3 juillet 1987, Paris, 1990, p. 37-47.

21. Z, 3, 1029a24. 
noté Burnyeat, à un lecteur intelligent, bien averti de ce qui se passe dans les traités de philosophie première et sans doute bon connaisseur de Z. Un tel lecteur ne peut que s'arrêter sur ce passage, éprouvant sans doute un moment de perplexité, et chercher ce qu'Aristote peut bien vouloir dire ici. C'est aussi une annonce, car la formule appelle des développements complémentaires, ceux-là mêmes que l'on va trouver en $H$, sur l'importance des types et des niveaux de détermination matérielle dans la définition des substances sensibles, et sur ce que veut dire « être en puissance ».

$\mathrm{H} 1$ propose en tout cas une conception de la matière - et corrélativement de la substance - beaucoup moins exclusive que celle de Z3. Or, si cette souplesse inattendue ne signifie pas changement de doctrine, elle est l'indice d'un changement de perspective ou d'objet à l'intérieur de l'enquête sur la substance. La matière, dans le texte [5], est comprise comme substance, parce qu'elle est substrat du changement, et cela sous ses quatre catégories, c'est-à-dire selon le lieu, la quantité, la qualité et la substance. Le texte reprend d'ailleurs sur ce dernier point Z, 7, 1032a1415. La nouvelle orientation de la discussion se confirme : il s'agit de dresser le cadre d'une investigation sur les substances en devenir; or ce cadre impose une prise en compte des propriétés de la matière en tant que sujet du changement.

Dans la suite du livre $\mathrm{H}$, l'intérêt pour la matière, loin de faiblir, se traduit par des efforts renouvelés pour en élaborer une conception plus fine. Cela commence par une rectification de la position de Démocrite, en $\mathrm{H}, 2$, 1042b11-18. Aristote reprend ici une description standard de la théorie démocritéenne des différences atomiques, en attribuant à l'Abdéritain un dispositif étiologique qu'il évoque également en A, 4, 985b4-20. Il procède à une sorte de traduction des trois termes utilisés par l'atomiste pour caractériser les différents types d'agrégations d'atomes (rhusmos, tropê, diathigê), et les transpose dans un lexique sans doute plus compréhensible à ses contemporains immédiats (schêma, thesis, taxis):

[6] Démocrite, pour sa part, semble penser qu'il y a trois différences - le corps servant de sujet, c'est-à-dire la matière, un et identique à lui-même, se différencie selon le rythme (rhusmos), qui est la configuration (schêma), ou selon l'orientation (tropê), qui est la position (thesis), ou selon l'arrangement (diathigê), qui est l'ordre (taxis). Les différences, cependant, sont manifestement en grand nombre, par exemple par juxtaposition des éléments matériels, par exemple par mixture, comme l'hydromel; ou encore par lien, comme un faisceau ; par collage, comme un livre (...).

Métaph., H, 2, 1042b11-18

Les différences sont chez Démocrite des caractéristiques de ce qu'Aristote appelle "matière », ou «corps sous-jacent». Elles sont en réalité, pour le Stagirite, des déterminations, sinon strictement «formelles », 
du moins « analogues à la substance » (1045a5). La raison en est simple : ces différences sont proprement les causes qui font que la chose est ce qu'elle est. Or c'est la substance qui est cause du fait qu'une chose est ce qu'elle est (1043a2-3). Ces causes ne sont pas matérielles, ainsi que le croient les physiciens, mais incorporelles, parce qu'elles sont simplement la manière dont les parties matérielles sont assemblées. La sunthesis, la juxtaposition des éléments matériels, introduit une différenciation (par collage, lien, mélange, etc.), différenciation elle-même immatérielle, qui explique pourquoi le composé se définit comme livre, faisceau ou hydromel. Ainsi, pour prendre un autre exemple donné dans la suite du texte, une même pierre peut être seuil ou linteau : c'est sa position, et non sa composition matérielle, qui la détermine essentiellement comme seuil ou linteau. Ainsi, bien que les différences mentionnées relèvent de catégories autres que l'ousia, nous sommes là devant des cas de prédications hylémorphiques et, de ce fait, face à des cas analogues à l'attribution d'une forme à une matière donnée.

L'affirmation selon laquelle c'est avant tout la forme qui est cause est un rappel implicite des acquis de $Z 17^{22}$. Mais il y a plus, ici. Les différences sont en effet considérées comme des actes (energeia : 1043a6), et ceux-ci sont prédiqués d'une matière donnée. De fait, les différences actualisent les potentialités contenues dans la matière qu'elles organisent :

[7] Il est clair d'après tout cela que l'acte est différent en différentes matières, et qu'il en va de même de la définition. Dans certains cas c'est la juxtaposition, dans d'autres le mélange, dans d'autres encore une autre $<$ des différences $>$ mentionnées. C'est pourquoi, parmi ceux qui donnent des définitions, certains, en disant que ce qu'est la maison, c'est « des pierres, des briques et du bois ", disent ce qu'est la maison en puissance, car c'est là sa matière ; mais d'autres, en proposant « un abri servant à protéger les biens et les personnes » ou quelque autre énoncé du même type, disent ce qu'est la maison en acte. Quant à ceux qui associent les deux, ils désignent le troisième type de substance, qui est composé de celles-ci (il semble en effet que la définition par les différences soit définition de la forme et de l'acte, et que celle qui énonce les composants internes, soit définition de la matière surtout).

Métaph., H, 2, 1043a12-21

Ce texte apporte trois éléments supplémentaires à l'analyse.

(i) En premier lieu, il rappelle le principe «autre forme, autre matière $»^{23}$. La matière de la glace est l'eau et non le son, aigu ou grave, qui est matière de l'harmonie. Il insiste sur les différences formelles (juxtaposition, mélange, etc.), mais la nature de la matière impliquée dans un composé donné n'est pas indifférente par rapport aux propriétés actuelles

22. Comme l'a souligné M. Burnyeat, op. cit., p. 73.

23. Phys., II, 2, 194 b9. 
de ce composé. La désignation de l'eau comme matière de la glace ne décrit d'ailleurs pas seulement les conditions de sa genèse (le processus de congélation), mais aussi ses propriétés actuelles (l'état de congélation). Cela signifie que la matière est en elle-même porteuse de déterminations bien précises et que les propriétés de la matière ne sont pas annulées dans la réalisation du composé mais conservées comme propriétés du composé lui-même.

(ii) Il n'en demeure pas moins, et c'est le second enseignement du passage, que l'existence des deux causes de déterminations que sont, d'une part la forme, d'autre part la matière, nous conduit à envisager différents types de définitions : une définition par les composants matériels (pour la maison: des pierres, des briques et du bois), une définition exclusivement formelle (un abri servant à protéger les biens et les personnes) et une définition comprenant les deux types déterminations (un abri fait de pierres, briques ou bois, servant à protéger les biens et les personnes). La première est définition de la maison selon la puissance puis selon les «composants internes » et vaut comme définition de ou par la matière; la seconde est définition selon l'acte puis selon «les différences » et vaut comme définition de ou par la forme et l'acte. C'est là admettre, comme par exemple en De anima, I, 1, 403a29-b16, à propos de la colère et des différentes définitions qu'elle est susceptible de recevoir, qu'il y a plusieurs manières de définir les substances composées et, de ce fait, que la matière est impliquée dans deux définitions sur les trois types de définitions envisagés. La suite du texte (1043a21-26) évoque les définitions élaborées ou admises par Archytas, qui mentionnaient à la fois la matière et la forme, par exemple la définition $\mathrm{du}$ vent calme comme un état de repos (forme ou acte) dans une masse d'air (matière ou puissance).

(iii) Le troisième enseignement de ce texte, sans doute le plus emblématique de la perspective adoptée en $\mathrm{H}$, c'est l'équivalence de la matière et de la puissance d'une part, de la forme et de l'acte d'autre part. Conformément à (i), cela suggère que, dans un composé donné (la maison), il faut comprendre à la fois - et non pas seulement successivement ou dans l'ordre de la génération - ce qui est acte et ce qui est puissance. Rien ne dit en effet que la première définition, celle qui ne mentionne que la matière, soit une formule exprimant un état antérieur à l'existence de la maison en acte, c'est-à-dire les pierres, les briques et les poutres disposées sur l'aire de chantier avant que la construction commence. Ce peut être aussi une définition, certes insuffisante, de ce que la maison est actuellement; de fait, la maison est actuellement - même si elle ne l'est pas exclusivement briques, pierres et poutres. Dans l'exemple donné par Aristote dans le De anima, celui de la colère, on voit bien que la définition par la seule matière (un « bouillonnement de sang dans la région du cœur») ne décrit pas un état antérieur à la colère elle-même, mais une modalité ou un aspect de la colère, qui existe actuellement, en même temps que le «désir de vengeance », qui constitue sa définition formelle. Ce point me paraît de première importance si l'on veut comprendre les deux thèses de H6, qui supposent la corrélation 
à la fois temporelle et ontologique de la puissance et de l'acte dans la substance composée ${ }^{24}$.

H4 apporte à son tour une contribution décisive à l'enquête sur la matière. On le voit en particulier dans le passage suivant, qui se trouve au début du chapitre :

[8] Concernant la substance matérielle, il ne doit pas échapper que, même si toutes choses viennent d'un même composant premier ou bien des mêmes composants premiers, et même si la même matière a fonction de principe pour les êtres en devenir, néanmoins il y en a une qui est appropriée à chacun. Par exemple : pour le phlegme, ce sont les composants sucrés ou gras; pour la bile, les composants amers ou autres. Peut-être toutefois ces composants viennent-ils du même composant. Par ailleurs, il y a plusieurs matières pour la même chose quand l'une est matière de l'autre. Par exemple : le phlegme vient du gras et du sucré, si le gras vient du sucré, et il vient de bile, par réduction de la bile à sa matière première. La relation « ceci vient de cela », en effet, s'entend en deux sens : ou bien au sens où il $\mathrm{y}$ a progression, ou bien au sens où il $\mathrm{y}$ a réduction au principe.

Métaph., H, 4, 1044a15-25

Ce passage est d'abord remarquable, on l'a vu, par l'usage de l'expression "substance matérielle », qui fait écho aux formules de H1. Il tient pour acquise l'existence d'une telle substance, mais les modalités de cette existence ne vont pas de soi. On a parlé précédemment de la substance entendue " comme substrat et matière » et de la substance sensible entendue en trois sens : comme matière, comme forme et acte et comme composée de celles-là (en H, 2, 1043a27-28). Il s'agit maintenant de comprendre comment se traduit, pour une substance sensible donnée, le fait d'avoir une matière.

Deux points sont à relever ici. Le premier est la distinction entre deux types ou deux niveaux de matière. Le premier niveau est celui du ou des composant(s) premier(s). Il ne s'agit sans doute pas d'une très hypothétique matière prime qui serait vierge de toute détermination, mais bien plus probablement de l'élément ou des éléments dont le composé provient. Or le composé ne provient pas de cette matière originelle en vertu de ses propriétés spécifiques, mais parce que toutes les choses qui lui sont matériellement homogènes en proviennent : "la même matière a fonction de principe pour les êtres en devenir ». On doit, pour caractériser la matière de telle chose particulière, donner sa matière propre ou "appropriée » (oikcía). Il faudra donc distinguer entre ces deux niveaux de matérialité, en vertu du principe «autre forme, autre matière » que l'on a vu à l'œuvre

24. Sur la question de la possibilité d'intégrer la matière dans la définition, outre mon commentaire déjà cité, p. 117-125, je renvoie à la synthèse récente et nuancée de R. Chiaradonna, " La chair et le bronze. Remarques sur Métaphysique Z, 11 et l'interprétation de M. Frede et G. Patzig », Les Études Philosophiques, 2014-3, p. 375-388. 
dans le texte [7]. Il est bien clair en tout cas que, du point de vue d'Aristote et contrairement à ce qu'ont affirmé la plupart des physiciens présocratiques, la matière qui compte dans l'explication causale - et qui doit être invoquée dans une définition comprenant à la fois la forme et la matière - est la matière la plus appropriée. Cela suppose que la matière soit considérée comme déjà informée; suffisamment informée en tout cas pour être la matière ultime ou prochaine, c'est-à-dire la plus appropriée à un composé donné.

Le second point qui me semble ici décisif est le suivant: la relation « ceci vient de cela », qu'on appellera « relation de provenance », n'est pas univoque. Elle se dit au moins en deux sens $(\delta \chi \chi \tilde{\omega} \varsigma)$. Ceci peut venir de cela au sens où il en est composé, comme le phlegme vient du gras. Il peut venir d'une autre matière encore par transitivité, comme le phlegme vient du doux, via le gras, qui vient du doux. Dans ce cas, il y a relation de composition, relation elle-même décomposable puisqu'il peut y avoir différents niveaux de composition. Toutefois, la relation de provenance ne se réduit pas à la relation de composition matérielle, puisque je peux dire que le phlegme vient de la bile, par réduction au principe matériel, alors que ce n'est pas la bile qui est composant du phlegme, mais l'inverse. La matière élémentaire peut donc remplir deux fonctions distinctes dans l'explication ${ }^{25}$ : celle du terminus a quo, dans le processus de progression à partir des composants de base ; celle de terminus ad quem, dans le processus analytique ou régressif.

Le chapitre 5 prolonge la réflexion engagée au chapitre 4 concernant les différentes façons de prendre en compte la matière dans l'explication. Il montre globalement, que, s'il est vrai que tout devenir implique les contraires, tous les contraires ne viennent pas à proprement parler les uns des autres - ce n'est pas le blanc lui-même qui devient à partir du noir, mais c'est le bois qui devient blanc - et que, dans le cas des êtres soumis au devenir, leur matière n'est pas toujours dans le même rapport avec les contraires. Tout corps dont provient un autre corps n'est pas nécessairement «matière » de ce dernier. Ainsi, je peux dire que le vinaigre vient du vin, mais je ne peux pas dire - du moins pas en toute rigueur - que le vin est matière du vinaigre. Je peux dire que le cadavre vient du vivant, mais pas que le vivant est matière du cadavre. La provenance est dans ce cas purement accidentelle. L'animal, c'est-à-dire le composé âme-corps, n'est pas matière du cadavre, parce qu'il n'a pas pour potentialité naturelle en tant qu'animal de devenir cadavre. En revanche, le corps de l'animal est plus

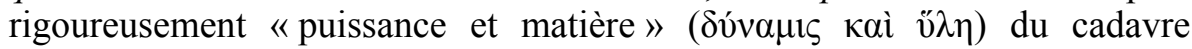
(1045a2), de même que l'eau peut être indifféremment vin ou vinaigre sans qu'aucune de ces deux réalisations soit contraire à sa nature.

25. Pour un réexamen récent du livre $\mathrm{H}$ à la lumière de la question de la matière et de son rôle dans l'explication, voir S.G. SEmINARA, Matter and Explanation. On Aristotle's Metaphysics Book H, Thèse, École normale supérieure de Lyon - Università degli studi Roma Tre. Dipartimento di filosofia, 2014. 
Le chapitre 5 précise donc les conditions de l'explication par la matière : il est vrai qu'il faut toujours privilégier la matière prochaine, ainsi que le chapitre 4 l'a recommandé. Cela ne suffit pas, cependant, à expliquer toutes les formes de provenances. Il faut en outre, dans certains cas, faire appel à une matière plus éloignée, comme la matière élémentaire. La matière propre ou prochaine ne suffit donc pas à expliquer toute forme de devenir. La méthode de définition et d'explication des composés hylémorphiques et de leurs propriétés passe en tout cas par une conception raffinée de la matière, conception dont les chapitres 4 et 5 dessinent les grandes lignes, ce qui n'est fait nulle part ailleurs dans la Métaphysique.

$$
* * *
$$

On voit donc que les deux thèses de H6 (la double équivalence de la matière et de la puissance et de la forme et de l'acte; l'unité immédiate du composé) sont préparées dans les chapitres précédents ${ }^{26}$. Il y a bien, dans le livre $\mathrm{H}$, un mouvement de fond en direction d'une conception plus fine de la matière, qui n'est plus simplement un substrat, mais un ensemble de potentialités.

Ceux-ci montrent en effet que :

- la matière elle aussi est en un sens substance ;

- la matière n'est pas dépourvue de déterminations, ce qui justifie notamment l'application du principe «autre forme, autre matière »;

- elle entre légitimement dans la définition des substances composées, sinon dans tous les cas, du moins sous certaines conditions ;

- la matière peut jouer divers rôles dans l'explication : non seulement par progression du composant vers le tout ultime, mais aussi par réduction ou résolution du tout ultime vers ses composants ;

- les notions de puissance et de matière sont équivalentes ;

- la puissance et la matière ne sont pas seulement l'état originaire de la réalité en acte, mais également un aspect constitutif de son état actuel ;

- les déterminations de la matière - et corrélativement de la puissance - sont graduelles.

26. Ce chapitre mérite assurément pour lui-même une analyse que je ne peux livrer ici. Je renvoie notamment à mon commentaire déjà cité, p. 41-59 et 185-205, et à : V. HARTE, «Metaphysics H6: A Dialectic with Platonism », Phronesis 41, 1996-3, p. 276-304; E. KeELING, «Unity in Aristotle's Metaphysics H6», Apeiron 45, 2012, p. 238-261; S. Delcomminette, « Métaphysique H 6 : unité de l'ousia, unité de l'eidos », Elenchos XXV, 2014-1, p. 89-125. 
En vertu de cette dernière proposition, on doit admettre qu'il n'y a pas d'hétérogénéité radicale de la matière - en particulier de la matière ultime et de la détermination formelle. H6 franchit un pas de plus, en affirmant que ce qu'une chose est sous l'aspect de sa matière ultime ou prochaine - par opposition à la matière non différenciée ou identifiable à des niveaux plus éloignés - ne diffère pas substantiellement de ce qu'elle est sous l'aspect de sa forme. Non seulement ce qui est informé ne peut exister sans ce qui informe, et réciproquement, mais encore la matière la plus élaborée ne diffère de la forme elle-même que par le fait d'être en puissance. Le gradualisme matériel, articulé à la distinction modale de la puissance et de l'acte, permet de comprendre que matière et forme ne s'additionnent pas comme des composants de même régime et qu'elles réalisent immédiatement, par elles-mêmes, l'unité de ce qu'elles composent. Il n'y a pas de tiers principe, en plus de la matière et de la forme, qui interviendrait dans l'unification de la substance composée. Du même coup, on comprend mieux que la matière puisse être dite ousia d'une chose déterminée : elle n'est pas par elle-même substance, mais elle ne fait qu'un in re avec la forme dans la constitution de la substance composée.

Je ne sais pas si la double affirmation de H6 - les textes [2] et [3] s'explique intégralement par les thèses préparatoires que j'ai identifiées dans les chapitres antérieurs du livre $\mathrm{H}$. On considère souvent que l'affirmation de l'unité du composé à la fin de H6 est proposée comme une solution au problème de l'unité de la définition et plus généralement de la prédication : l'unité de " animal-bipède », qui fait problème si l'on accepte la doctrine platonicienne de la participation, doit en fait être conçue par analogie avec l'unité de la matière et de la forme, dont la nature même est d'être corrélées et parfaitement unifiées, comme le sont par exemple l'âme et le corps. Or ce problème est déjà posé au livre $\mathrm{Z}$, en particulier en $\mathrm{Z} 12^{27}$. Le parcours que l'on vient d'effectuer montre en tout cas que la solution hylémorphiste de H6 au problème de l'unité des substances sensibles présuppose le travail antérieurement mené en $\mathrm{H}$, en particulier parce qu'elle appelle une conception raffinée de la matière et de la causalité matérielle.

L'étude de la matière a donc bien sa place dans les traités métaphysiques. Elle ne l'a pas pour elle-même : elle relèverait dans ce cas de la physique, science des composés de matière et de forme en tant qu'ils sont affectés par le changement. Toutefois, subordonnée à l'enquête sur la substance en tant que telle - qui incombe à la philosophie première -, une telle investigation se justifie à un double titre. On doit d'abord admettre que la matière, parce qu'elle est sujet du changement et constitue un «ceci» en puissance, a part à la substantialité d'une certaine manière, et qu'en ce sens précis « la matière également est substance ». La matière, en corrélation fonctionnelle avec la forme, œuvre directement à faire de la substance

27. Par ailleurs, pour être tout à fait complet sur le statut de la matière en H6, il conviendrait d'analyser la matière « intelligible » ou «pour la raison », évoquée en 1045a33-b7. Je m'en tiens toutefois ici à la seule matière « sensible ». 
composée, précisément, une substance. Lorsqu'il s'agit, ensuite, de comprendre le statut propre et l'unité de la substance composée, il faut pouvoir expliquer comment la matière se rapporte à la forme sans pour autant que l'une s'additionne à l'autre. Prétendre que cette préoccupation est étrangère à la philosophie première reviendrait à occulter la perspective ouverte par Z17, et notamment par l'indication de 1041b5-9, selon laquelle la question causale proprement essentielle, ou substantielle, revient à chercher pourquoi une matière donnée est telle ou telle chose, et à identifier pour cela la forme. On comprend donc que la forme est cause éminente, mais également que, si elle est telle, dans les substances composées, c'est en fonction de la matière qu'elle informe. Dans cette optique, l'explicitation au livre $\mathrm{H}$ du rapport matière-forme en termes de puissance et d'acte - parce que ce modèle, articulant deux modes d'êtres qui ne s'additionnent pas l'un à l'autre mais sont fonctionnellement corrélés, parvient à rendre compte de l'unité substantielle - joue un rôle essentiel. C'est donc dans le livre H, mieux qu'en aucun autre des traités métaphysiques, que l'on peut s'attendre à trouver les principes d'une telle investigation, parce que c'est là que la solution de l'unité de la substance en termes d'unité hylémorphique trouve son expression la plus claire. 